\title{
Bilateral Renal Artery Stenosis With a Pheochromocytoma
}

\author{
Benjamin Chaucer ${ }^{\mathrm{a}, \mathrm{c}}$, Fidencio Davalos ${ }^{\mathrm{b}}$, Wahib Zafar ${ }^{\mathrm{b}}$, Jamila Benmoussa ${ }^{\mathrm{b}}$, \\ Farhang Ebrahimi ${ }^{\mathrm{b}}$, Jay Nfonoyim ${ }^{\mathrm{b}}$
}

\begin{abstract}
Renal artery stenosis (RAS) is a common cause of secondary hypertension. Pheochromocytomas have been associated with hypertension in $0.1 \%$ of the population. Pheochromocytoma is a well-documented co-morbid condition of unilateral RAS. Bilateral RAS with a pheochromocytoma is extremely rare with only one documented case in the literature. We present the second documented case of bilateral RAS with a pheochromocytoma. The coexistence of these pathologies makes diagnosis challenging. Proper diagnosis of the etiology of hypertension is essential given the pre-surgical treatment required for neuroendocrine tumors.
\end{abstract}

Keywords: Renal artery stenosis; Pheochromocytoma; Bilateral

\section{Introduction}

Unilateral renal artery stenosis (RAS) is a common cause of secondary hypertension. This incidence increases in the setting of atherosclerotic disease. Bilateral RAS is far less common. Pheochromocytoma is a neuroendocrine tumor of the adrenal medulla and results in increased blood pressure. In the general population, it is estimated that it may represent the cause of up to $0.1 \%$ of hypertension. The existence of a neuroendocrine tumor with unilateral RAS is well documented and may be the result of localized hormonal effect. The presence of bilateral RAS, however, is exceedingly rare with only one previous documented case in the literature.

\section{Case Report}

A 65-year-old man with a past medical history of HLD, DM2

Manuscript accepted for publication March 24, 2016

${ }^{a}$ St. Georges University School Medicine, 3500 Sunrise Hwy, Great River, NY 11739, USA

bichmond University Medical Center, 355 Bard Avenue, Staten Island, NY 10301, USA

${ }^{\mathrm{c} C o r r e s p o n d i n g ~ A u t h o r: ~ B e n j a m i n ~ C h a u c e r, ~ S t . ~ G e o r g e s ~ U n i v e r s i t y ~ S c h o o l ~}$ Medicine, 3500 Sunrise Hwy, Great River, NY 11739, USA.

Email: benchaucer@gmail.com

doi: http://dx.doi.org/10.14740/jem335w and HTN presented to the emergency department (ED) from his primary medical doctor after he was found to have a blood pressure of $210 / 120 \mathrm{~mm} \mathrm{Hg}$. In the ED, his blood pressure measured while lying was 143/70 mm Hg. He was given benicar and amlodipine 5 and $40 \mathrm{mg}$, respectively. The patient reported having recently started taking metoprolol. After interviewing the patient's wife, she stated that after starting the new medication, he suddenly began forgetting things, asking repetitive questions, and experiencing sweating with associated dizziness. The patient conceded that he was noncomplaint with taking regular POC glucose levels for the past year. Family history was positive for one sister with a history of diabetes type II. The patient had no history of smoking or drug use and admitted to occasional alcohol consumption on the weekends. Review of systems was positive for fatigue and weakness and negative for chills, or changes in weight. He reported lightheadedness with no exertional dyspnea, edema, cough, abdominal pain, constipation, diarrhea or hematochezia. The patient stated that he had a headache of 1 week duration with nausea and vomiting 2 days prior to admission. Laboratory data in the ER showed a white blood cell count of 11.5 , hemoglobin of 16.5 , creatinine of 2.3 , BUN of 33 , potassium of 6.8 , sodium of 136 and bedside glucose of 133 . He was treated with IV insulin, calcium gluconate, and D50. Repeat vitals showed that the patient had a blood pressure of 127/76 mm Hg. Cardiovascular exam showed regular rate, and rhythm with no jugular venous distention or murmur. Auscultation of the abdomen revealed normal bowel sounds, with no tenderness in all four quadrants to light and deep palpation. The patient had $2+$ pulses in all extremities bilaterally without edema. Renal artery Doppler was performed to assess the causes of hypertension. Bilaterally elevated velocities of the renal arteries were discovered consistent with a 50\% stenosis bilaterally. Computed tomography was performed and found a heterogeneous left adrenal mass measuring $6.3 \mathrm{~cm}$ $($ cephalocaudal $) \times 5.9 \mathrm{~cm}($ transverse $) \times 6 \mathrm{~cm}(\mathrm{AP})$. The mass was well circumscribed and did not invade the surrounding tissues. A diagnosis of pheochromocytoma was made. The 24-h urine catecholamines were ordered and showed plasma normetanephrine of 3,588, and total plasma metanephrine of 3,640 . Total catecholamine level was 10,755 with dopamine of 76, epinephrine of 63 and norepinephrine of 10,692. The patient was started on doxazosin $2 \mathrm{mg}$ once a day initially then was increased to $1 \mathrm{mg}$ in the morning and $2 \mathrm{mg}$ at night. The patient was discharged home after symptomatic resolution with a 1 -week follow-up with his primary medical doctor. 


\section{Discussion}

RAS is a common cause of secondary hypertension. Incidence is not clearly defined but some studies suggest that it may be as high as $0.5-5 \%$ in patients with secondary hypertension [1]. Bilateral RAS is much more rare. Duke University performed a study, in which 3,987 patients underwent abdominal aortography to screen for RAS and found that $4.8 \%$ had stenosis of $75 \%$ or greater with only $0.8 \%$ having bilateral RAS [2]. The severity of the occlusion correlates directly to patient survival with bilateral RAS having a 2-year survival rate of $74 \%$ compared with unilateral RAS with a survival rate of $96 \%$ [3]. RAS results in decreased perfusion to the kidney and decreased glomerular filtration rate (GFR). The decrease in GFR results in increased release of renin by the juxtaglomerular cells. Renin converts angiotensinogen to angiotensin I which is converted by angiotensin-converting enzyme (ACE) resulting in the formation of angiotensin II. Angiotensin II exerts systemic effects resulting in increased blood pressure. RAS is commonly seen in the setting of atherosclerotic disease. Neurofibromatosis type 1 (NF1) and unilateral RAS is a well-documented phenomenon. Unilateral RAS is associated with diseases such as NF1, Kawasaki disease, congenital rubella, Crohn's disease and Marfans syndrome [4]. A literature search showed only one case of bilateral RAS associated with NF1 [5]. Our patient lacked the diagnostic criteria necessary for NF1 making his diagnosis of bilateral RAS with a pheochromocytoma exceedingly rare. While the presence of a pheochromocytoma with unilateral RAS has been well documented, there is only one case of bilateral RAS with a pheochromocytoma [5]. Incidence of pheochromocytoma with unilateral RAS may be as high as 3.4-7.0\%, although this number is yet to be clearly defined $[6,7]$. Pheochromocytomas are derived from the chromaffin cells of the adrenal medulla. They secrete catecholamine, which result in systemic effects such as increased blood pressure, tachycardia, panic and cyclical diaphoresis. The management of patients with pheochromocytomas differs substantially when compared to the management of patients with uncomplicated RAS. While surgical treatment is not recommended for RAS with $<70 \%$ stenosis, it is the treatment of choice for pheochromocytoma. Burns et al suggest that the presence of RAS may be due to localized hormone effects from the pheochromocytoma [8]. This hypothesis is made based on the finding that many cases of RAS are found on the same side as the pheochromocytoma. This fails however to explain the correlation between bilateral RAS and the presence of a single pheochromocytoma.
The diagnosis of such cases is challenging given that both diseases result in an elevation of blood pressure. The diagnosis of pheochromocytoma is made by collection of 24-h urine fractioned metanephrines and catecholamines. Elevation in biochemical tests should result in imaging confirmation. RAS is diagnosed first by evaluating serum renin and creatinine to assess renal function and perfusion. Confirmation can be performed by renal Doppler or MRI/CT scan. Given the high incidence of pheochromocytoma in RAS, patients found with an elevation in blood pressure may benefit from monitoring of both pathologies. This is particularly important given the extensive pre-operative treatment required for surgical removal of pheochromocytomas.

\section{References}

1. Simon N, Franklin SS, Bleifer KH, Maxwell MH. Clinical characteristics of renovascular hypertension. JAMA. 1972;220(9):1209-1218.

2. Harding MB, Smith LR, Himmelstein SI, Harrison K, Phillips HR, Schwab SJ, Hermiller JB, et al. Renal artery stenosis: prevalence and associated risk factors in patients undergoing routine cardiac catheterization. J Am Soc Nephrol. 1992;2(11):1608-1616.

3. Mailloux LU, Napolitano B, Bellucci AG, Vernace M, Wilkes BM, Mossey RT. Renal vascular disease causing end-stage renal disease, incidence, clinical correlates, and outcomes: a 20-year clinical experience. Am J Kidney Dis. 1994;24(4):622-629.

4. Daniels SR, Loggie JM, McEnery PT, Towbin RB. Clinical spectrum of intrinsic renovascular hypertension in children. Pediatrics. 1987;80(5):698-704.

5. Anjum Muhammad Sohail. Bilateral Renal Artery Stenosis and Pheochromocytoma an Uncommon Association: A Case Report. International Journal of Medicine and Public Health. 2014:4(4):517.

6. Gill IS, Meraney AM, Bravo EL, Novick AC. Pheochromocytoma coexisting with renal artery lesions. J Urol. 2000;164(2):296-301.

7. Sarathi V, Bandgar T, Lila AR, Deshpande AA, Dalvi AN, Patwardhan S, Shah NS. Coexistence of pheochromocytoma/praganglioma and renal artery stenosis. Indian J Endocrinol Metab. 2012;16(6):1009-1011.

8. Burns AP, O'Connell PR, Murnaghan DJ, Brady MP. Bilateral adrenal phaeochromocytomas associated with unilateral renal artery stenosis. Postgrad Med J. 1989;65(770):943-947. 